\title{
ON DIGITAL DISTRIBUTION IN SOME INTEGER SEQUENGES
}

B. D. CRAVEN

(Received 7 September 1964, revised 15 January 1965)

\section{Introduction}

Although the harmonic series

$$
1+\frac{1}{2}+\frac{1}{3}+\frac{1}{4}+\cdots
$$

diverges, there is a sense in which it "nearly converges". Let $N$ denote the set of all positive integers, and $S$ a subset of $N$. Then there are various sequences $S$ for which

$$
T=\sum_{n \in S} \frac{1}{n}
$$

converges, but for which the "omitted sequence" $N-S$ is, in an intuitive sense, sparse, compared with $N$. For example, Apostol [1] (page 384) quotes, without proof, the case where $S$ is the set of all positive integers whose decimal representation does not involve the digit zero (e.g. $7 \in S$ but $101 \notin S$ ); then (1) converges, with $T<90$.

It is shown in this paper that Apostol's example is a special case of a general theorem on a class of sequences $S$ for which $T$ converges. From this it follows that certain integer sequences - in particular the sequence of prime numbers - include, for each integer $d$, a term whose representation to a given base contains any given digit at least $d$ times. For example, there exists a prime $p$ whose decimal representation contains at least 100 zeros. Although the existence proof for $p$ is not constructive, an asymptotic bound for $p$ is obtained, using the prime number theorem of Hadamard and de la Vallée Poussin.

\section{Harmonically convergent sequences}

An increasing sequence of positive integers $\left\{n_{1}, n_{2}, \cdots\right\}$, for which the series of reciprocals

$$
\frac{1}{n_{1}}+\frac{1}{n_{2}}+\frac{1}{n_{3}}+\cdots
$$


converges, will be called "harmonically convergent". The sum of the series (2) will then be called the "harmonic sum" of the sequence. A sequence for which the sum of reciprocals (2) diverges will be called "harmonically divergent".

A large class of harmonically convergent sequences is characterised by the following theorem.

Theorem 1. For integers $b=2,3,4, \cdots, d=1,2,3, \cdots, t=0,1$, $2, \cdots, b-1$, let $S(b, d, t)$ denote the increasing sequence of all positive integers whose representation to base $b$ involves the digit $t$ at most $(d-1)$ times. Then $S(b, d, t)$ is harmonically convergent, and its harmonic sum is (strictly) less than $b^{d}(1+d \log b)$.

Proof. For each positive integer $r$, denote by $D(r)$ the set of $b^{d}$ consecutive integers whose least member is $b^{d} r$.

If $r \notin S(b, d, t)$, then $D(r)$ contains no members of $S(b, d, t)$.

If $r \in S(b, d, t)$, then $D(r)$ contains at most $\left(b^{d}-1\right)$ members of $S(b, d, t)$, since one member of $D(r)$ has the digit $t$ in each of its last $d$ positions. Let $C(r)$ denote the sum of the reciprocals of these at most $\left(b^{d}-1\right)$ integers. Then, for $r \in S(b, d, t), C(r)$ is (strictly) less than $\left(b^{d}-1\right)\left(b^{d} r\right)^{-1}$. In particular, if $r_{0}$ is the least member of $S(b, d, t)$, then

Denote also

$$
\Delta \equiv\left(b^{d}-1\right)\left(b^{d} r_{0}\right)^{-1}-C\left(r_{0}\right)>0 .
$$

$$
C(0)=\frac{1}{1}+\frac{1}{2}+\frac{1}{3}+\cdots+\frac{1}{b^{d}-1} .
$$

Let $T_{q}$ denote the sum of the reciprocals of the first $q$ members of $S(b, d, t)$. Let $\sum_{r}^{\prime}$ denote summation only over values of $r$ which belong to $S(b, d, t)$. Then, for all $q>\left(r_{0}+1\right) b^{d}$,

$$
T_{a}<C(0)+\sum_{r=1}^{q} C(r)
$$

since the right side includes all terms of $T_{q}$, plus additional positive terms. Therefore

so that

$$
T_{q}<\left\{1+\log \left(b^{d}-1\right)\right\}+\left\{\left(b^{d}-1\right) b^{-d} T_{q}-\Delta\right\}
$$

$$
T_{q}<b^{d}(1+d \log b-\Delta) \text {. }
$$

Hence $S(b, d, t)$ is harmonically convergent, and its harmonic sum $T(b, d, t)$ satisfies the inequality

$$
T(b, d, t)<b^{d}(1+d \log b) .
$$




\section{Harmonically divergent sequences}

Let $W=\left\{n_{1}, n_{2}, \cdots\right\}$ denote any harmonically divergent sequence. For $j=1,2,3, \cdots$, define the functions

$$
\begin{aligned}
& G(j)=\frac{1}{n_{1}}+\frac{1}{n_{2}}+\cdots+\frac{1}{n_{j}} \\
& H(j)=\frac{1}{n_{1}}+\frac{1}{n_{2}}+\cdots+\frac{1}{n_{r}}
\end{aligned}
$$

where $n_{r}$ is the largest member of $W$ not exceeding $j$.

Since $G(j)$ and $H(j)$ are increasing functions, their inverses $G^{-1}(x)$ and $H^{-1}(x)$ are defined for values of $x$ which fall in the ranges of $G$ and $H$ respectively. For values of $x$ which do not, let $x^{\prime}$ denote the largest number, not exceeding $x$, which lies in the range of $G$; then define $G^{-1}(x)=G^{-1}\left(x^{\prime}\right)$. Similarly define $H^{-1}(x)$.

THEOREM 2. Let $W$ be a harmonically divergent sequence. Let $b \geqq 2$ and $d \geqq 1$ be integers. Then for every choice of the integers $b^{\prime}=2,3, \cdots, b$ and $t=0,1,2, \cdots, b^{\prime}-1$, there is a member $n_{i}=n_{i}(b, d, t)$ of $W$ rohose representation to base $b^{\prime}$ contains the digit $t$ at least $d$ times, and such that

$$
\begin{aligned}
n_{i} & \leqq H^{-1}(T(b, d, t)) \\
i & \leqq G^{-1}(T(b, d, t)) .
\end{aligned}
$$

Proof. Let $b, d, t$ be given. Then by Theorem $1, W$ is not contained in the set $S(b, d, t)$, so $W$ includes an integer $n$ whose representation to base $b$ contains the digit $t$ at least $d$ times. Again by Theorem 1, a partial sum (3) which exceeds $T(b, d, t)$ must contain such a number $n$, so (5) is proved, for $b^{\prime}=b$. A similar proof applies to (6).

For $b^{\prime}<b$, (5) and (6) thus hold, if $b^{\prime}$ replaces $b$. Now if $t$ and $d$ are given, $T(b, d, t)$ increases as $b$ increases, because an increase of $b$ replaces each integer in the "omitted sequence" by a greater integer. So (5) and (6) hold as stated, since $H^{-1}$ and $G^{-1}$ are increasing functions.

If $b^{\prime}=b$, the bounds (5) and (6) are "best possible", in the sense that for any positive $\varepsilon$, there is a harmonically divergent sequence for which

and

$$
n_{i}>H^{-1}(T(b, d, t)-\varepsilon)
$$

$$
i>G^{-1}(T(b, d, t)-\varepsilon) \text {. }
$$

It suffices to take a harmonically divergent sequence $W$ which contains only terms of $S(b, d, t)$, until the partial sum of the series of reciprocals exceeds $T(b, d, t)-\varepsilon$. 
Combining (5) and (6) with the bound of Theorem 1 proves the Corollary. With symbols as in Theorem 2,

$$
\begin{aligned}
n_{i} & \leqq H^{-1}\left(b^{d}(1+d \log b)\right) \\
i & \leqq G^{-1}\left(b^{d}(1+d \log b)\right) .
\end{aligned}
$$

\section{Applications}

As one application of theorem 2 , let $\alpha$ and $c$ satisfy $0<\alpha<1$ and $c>0$. Then the sequence $\left\{n_{j}\right\}$, where $n_{j}=\left[c j^{\alpha}\right]$, and $[x]$ denotes "greatest integer $\leqq x^{\prime \prime}$, is harmonically divergent. For this sequence,

so by (8),

$$
G(j) \geqq \int_{1}^{j+1} \frac{d x}{c x^{\alpha}}=\frac{(j+1)^{1-\alpha}-1}{c(1-\alpha)} ;
$$

$$
i \leqq\left\{1+c(1-\alpha) b^{\alpha}(1+d \log b)\right\}^{1 /(1-a)}-1 .
$$

Thus, for example, for every choice of $b^{\prime} \leqq 10$ and $0 \leqq t<b$, there is an integer $n<4.3 \times 10^{14}$ (approx.), such that the representation of [ $\left.\pi n \pm\right]$ to base $b^{\prime}$ contains digit $t$ at least 6 times.

Similar conclusions apply to the sequences $\{[c \log j]\}$ and $\{[c j \log j]\}$, where $j=1,2,3, \cdots$.

Let $P$ denote the sequence of prime numbers $\left\{p_{1}, p_{2}, \cdots\right\}$. Let $P_{a \beta}$ denote the subsequence $\left\{P_{\alpha+n \beta}: h=0,1,2, \cdots\right\}$, for given integers $\alpha$ and $\beta$. It is well known (e.g. [2]) that $P_{\alpha \beta}$ is harmonically divergent, therefore Theorem 2 applies to $P_{\alpha \beta}$. To approximate to the bounds (7) and (8), let $a(n)=1$ when $n \in P_{\alpha \beta}, a(n)=0$ otherwise; let $A(n)=a(1)+a(2)+\cdots$ $+a(n)$; then $A(n)$ equals the number of primes in $P_{\alpha \beta}$ which do not exceed $n$. Then for $P_{\alpha \beta}$,

$$
\begin{aligned}
H\left(2^{k}\right) & =\sum_{j=1}^{2^{k}} \frac{a(j)}{j}=\frac{A\left(2^{k}\right)}{2^{k}}+\sum_{1}^{2^{k}-1} A(j) \cdot\left(\frac{1}{j}-\frac{1}{j+1}\right) \\
& \geq \sum_{a=2}^{k} \sum_{j=2^{q-1}}^{2^{a}-1} A(j) \cdot\left(\frac{1}{j}-\frac{1}{j+1}\right) \\
& >\sum_{a=2}^{k} A\left(2^{q-1}\right) \cdot\left(\frac{1}{2^{q-1}}-\frac{1}{2^{q}}\right) \\
& =\frac{1}{2} \sum_{2}^{k} A\left(2^{a-1}\right) / 2^{a-1} .
\end{aligned}
$$


From the Prime Number Theorem, $A(n)$ is given asymptotically by

$$
A(n) \sim \frac{n}{\beta \log n} \quad \text { as } n \rightarrow \infty .
$$

Here the symbol $f(n) \sim g(n)$ means that

$$
\lim _{n \rightarrow \infty} f(n) / g(n)=1 .
$$

It will be convenient also to use the expressions " $f(n)$ is asymptotically less than $g(n)$ " or " $g(n)$ is asymptotically greater than $f(n)$ " to mean

$$
\limsup _{n \rightarrow \infty} f(n) / g(n) \leqq 1 .
$$

If this holds, then for any $\varepsilon>0, f(n)<(1+\varepsilon) g(n)$ for all $n$ sufficiently large; $g(n)$ may thus also be termed an "asymptotic upper bound to $f(n)$ ", as $n \rightarrow \infty$.

Now from (10), for any $\varepsilon>0$,

$$
\frac{A(n)}{n}>\frac{1-\varepsilon}{\beta \log n} \quad \text { for all } n>n(\varepsilon) \text {. }
$$

Therefore

$$
\frac{1}{2} \sum_{2}^{k} A\left(2^{q-1}\right) / 2^{q-1}>\frac{1-\varepsilon}{2 \beta} \sum_{2}^{k} \frac{1}{\log 2^{q-1}}+B
$$

where the constant $B$ represents the error arising from those terms in the summation to which inequality (11) does not apply; since the number of such terms depends on $\varepsilon$, but not on $k, B$ does not depend on $k$.

Now if $k=[\log n / \log 2]$, then, for $n$ sufficiently large,

Let

$$
\begin{aligned}
H(n) \geqq H\left(2^{k}\right) & >B+\frac{1-\varepsilon}{2 \beta \log 2} \sum_{2}^{k} \frac{1}{q-1} \\
& >B+\frac{(1-\varepsilon) \log ([\log n / \log 2])}{2 \beta \log 2} .
\end{aligned}
$$

$$
L(n)=\{\log (\log n / \log 2)\} /\{2 \beta \log 2\} .
$$

Then, from (12), for any $\varepsilon>0$,

$$
\limsup _{n \rightarrow \infty} L(n) / H(n) \leqq 1 /(1-\varepsilon) .
$$

Consequently, $H(n)$ is an asymptotically greater than $L(n)$, as $n \rightarrow \infty$. Therefore, from (7), an asymptotic upper bound $\bar{n}$ for $n_{i}$, as $d \rightarrow \infty$, is given by 


$$
2 \beta \log 2 \cdot\left(b^{d}(1+d \log b)\right)=\log (\log \bar{n} / \log 2) .
$$

Let $P_{\alpha \beta}^{*}$ denote the set of prime numbers obtained by selecting arbitrarily exactly one prime from each subset

$$
\left\{p_{\alpha+\hbar \beta}, p_{\alpha+h \beta+1}, \cdots, p_{\alpha+(h+1) \beta-1}\right\},
$$

where $h=0,1,2, \cdots$. Then $P_{\alpha \beta}^{*}$ is also harmonically divergent, and the same asymptotic estimates, including (13), apply to $P_{\alpha \beta}^{*}$ as to $P_{\alpha \beta}$.

Similar results apply also to primes in arithmetic progression. Let $y$ and $z$ be relatively prime integers. Let $Q$ denote the set of all primes $p \equiv z$ $(\bmod y)$. Then LeVeque $[3]$ shows that the number of primes in $Q$ which do not exceed $n$ is asymptotically

$$
\frac{1}{\phi(y)} \int_{2}^{n} \frac{d u}{\log u} \quad \text { as } n \rightarrow \infty,
$$

where $\phi(y)$ is Euler's function.

A similar discussion to that for $P_{\alpha \beta}$ then shows that $Q$ is harmonically divergent, and the asymptotic bounds (12) and (13) apply also to $Q$, with $\beta=\phi(y)$.

As a numerical illustration of (13), set $b=10$ and $d=100$. Then for any base $\leqq 10$, there exists a prime $p$ whose representation contains a given digit at least 100 times; and an upper bound $\bar{n}$ to $p$ is asymptotically estimated by

$$
\log _{10} \log _{10} \bar{n}=1.4 \times 10^{102}
$$

\section{Acknowledgement}

My thanks are due to the referee for some improvements in the presentation of this paper.

\section{References}

[1] Apostol, T. M., Mathematical Analysis. (Addison-Wesley, 1957).

[2] Landau, E., Handbuch der Lehre von der Verteilung der Primzahlen, Vol. I.

[3] LeVeque, W. J., Topics in Number Theory, Vol. II. (Addison-Wesley, 1956).

University of Melbourne 